\title{
Formation of Interfacial Janus Nanomicelles by Reactive Blending and their Compatibilization Effects on Immiscible Polymer Blends
}

\author{
Hengti Wang, ${ }^{1,2,3}$ Zhiang Fu, ${ }^{1}$ Wenyong Dong, ${ }^{1}$ Yongjin $\mathrm{Li},{ }^{* 1}$ Jingye $\mathrm{Li}^{2}$
}

1 College of Materials, Chemistry and Chemical Engineering, Hangzhou Normal University, No.

16 Xuelin Rd., Hangzhou, 310036, P. R. China

2 CAS Center for Excellent on TMRS Energy System, Shanghai Institute of Applied Physics,

Chinese Academy of Sciences, R. R. China

3 University of Chinese Academy of Sciences, Beijing, 100049, P. R. China

\section{Table of contents}

\begin{tabular}{|c|c|}
\hline Eq. S1 & $\begin{array}{l}\text { Synthesis of P(S-co-GMA) (RLC) by copolymerization of Styrene and Glycidyl } \\
\text { methacrylate }\end{array}$ \\
\hline Eq. S2 & $\begin{array}{l}\text { Synthesis of } \mathrm{P}((\mathrm{S}-\mathrm{co}-\mathrm{GMA})-\mathrm{g}-\mathrm{MMA})(\mathrm{RGC}) \text { through "Grafting through" method by } \\
\text { copolymerization of MMA-Macromer, Styrene and Glycidyl methacrylate }\end{array}$ \\
\hline Eq. S3 & $\begin{array}{l}\text { In-situ grafting reaction of } \mathrm{P}((\mathrm{S}-\text { co-GMA)- } g \text {-MMA) }(\mathrm{RGC}) \text { with PLLA within } \\
\text { reactive melt blending }\end{array}$ \\
\hline Fig. S1 & $\begin{array}{l}\text { Partial enlargement of strain-stress curves of neat PVDF, neat PLLA, pristine } \\
\text { PVDF/PLLA }(50 / 50, \mathrm{w} / \mathrm{w}) \text { blend and PVDF/PLLA blends with } 3 \mathrm{wt} \% \text { reactive } \\
\text { copolymers of various topological structures }\end{array}$ \\
\hline Tab. S1 & $\begin{array}{l}\text { Mechanical properties of neat PVDF, neat PLLA, pristine PVDF/PLLA }(50 / 50, \mathrm{w} / \mathrm{w}) \\
\text { blend and JNMs compatibilized PVDF/PLLA blends with } 3 \mathrm{wt} \% \text { reactive } \\
\text { copolymers of various topological structures }\end{array}$ \\
\hline Fig. S2 & $\begin{array}{l}\text { Partial enlargement of stress-strain curves of JNMs compatibilized } \\
\text { PVDF/PLLA/RGC (50/50/3, w/w/w) blends with various epoxy content: RGC-0, } \\
\text { RGC-5, RGC-10 and RGC-20 represented epoxy of } 0 \mathrm{wt} \%, 5 \mathrm{wt} \%, 10 \mathrm{wt} \% \text { and } 20\end{array}$ \\
\hline
\end{tabular}




\begin{tabular}{|c|c|}
\hline & $\mathrm{wt} \%$ in $\mathrm{RGC}$, respectively \\
\hline Fig. S3 & $\begin{array}{l}\text { The TEM statistical analysis of nanomicelles at the PVDF-PLLA interface of its } \\
\text { compatibilized PVDF/PLLA (50/50, w/w) blends with various epoxy content: } \\
\text { RGC-0, RGC-5, RGC-10 and RGC-20 represented epoxy of } 0 \mathrm{wt} \%, 5 \mathrm{wt} \%, 10 \mathrm{wt} \% \\
\text { and } 20 \mathrm{wt} \% \text { in RGC, respectively }\end{array}$ \\
\hline Tab. S2 & $\begin{array}{l}\text { Thermal parameters and mechanical properties of JNMs compatibilized } \\
\text { PVDF/PLLA/RGC (50/50/3, w/w/w) blends with various epoxy contents in RGC }\end{array}$ \\
\hline Fig. S4 & $\begin{array}{l}\text { Figure S4. Partial enlargement of stress-strain curves of JNMs compatibilized } \\
\text { PVDF/PLLA }(50 / 50, \mathrm{w} / \mathrm{w}) \text { blends with various GRC-20 loadings }(0 \mathrm{wt} \%, 1 \mathrm{wt} \% \text {, } \\
3 \mathrm{wt} \% \text { and } 5 \mathrm{wt} \%)\end{array}$ \\
\hline Tab. S3 & $\begin{array}{l}\text { Mechanical properties of JNMs compatibilized PVDF/PLLA }(50 / 50, \mathrm{w} / \mathrm{w}) \text { blends } \\
\text { with various GRC-20 Loadings }(0 \mathrm{wt} \%, 1 \mathrm{wt} \%, 3 \mathrm{wt} \% \text { and } 5 \mathrm{wt} \%)\end{array}$ \\
\hline Fig. S5 & $\begin{array}{l}\text { Partial enlargement of stress-strain curves of PVDF/PLLA/RGC-20 (50/50/3, } \\
\text { w/w/w) blend with various mixing methods }\end{array}$ \\
\hline Tab. S4 & $\begin{array}{l}\text { Mechanical properties of JNMs compatibilized PVDF/PLLA/RGC-20 (50/50/3, } \\
\text { w/w/w) blends with various mixing methods }\end{array}$ \\
\hline Tab. S5 & Molecular parameters of components in blends \\
\hline
\end{tabular}




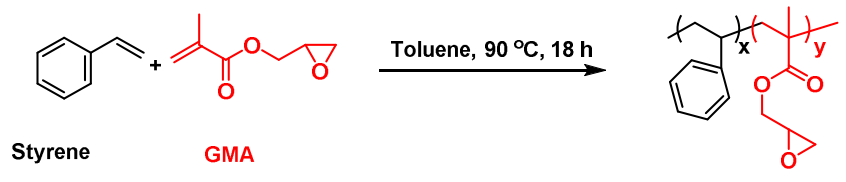

P(S-co-GMA)

Reactive Linear Copolymer (RLC)

Equation S1. Synthesis of P(S-co-GMA) (RLC) by copolymerization of Styrene and Glycidyl methacrylate 


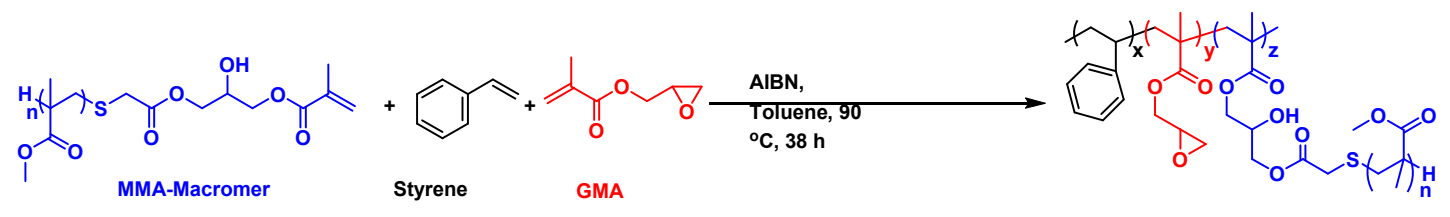

P((S-co-GMA)-g-MMA)

Reactive Graft Copolymer (RGC)

Equation S2. Synthesis of P((S-co-GMA)-g-MMA) (RGC) through "Grafting through" method by copolymerization of MMA-Macromer, Styrene and Glycidyl methacrylate 


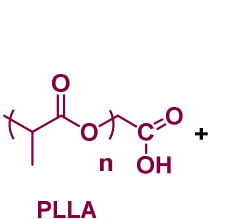

PLLA

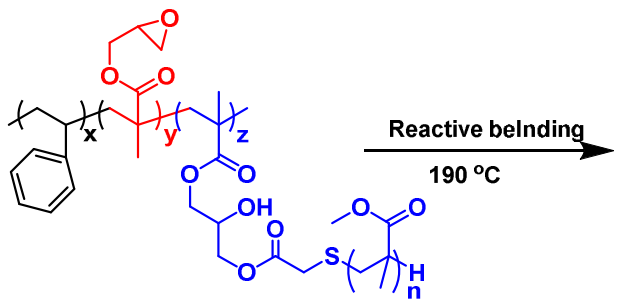

P((S-co-GMA)-g-MMA)

Reactive Graft Copolymer (RGC)

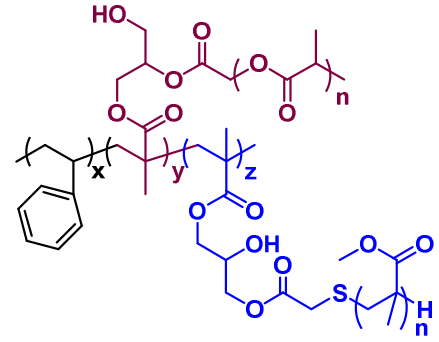

Dual-Graft Copolymer (DGC)

Equation S3. In-situ grafting reaction of $\mathrm{P}((\mathrm{S}-c o-\mathrm{GMA})-g$-MMA) (RGC) with PLLA within

reactive melt blending 


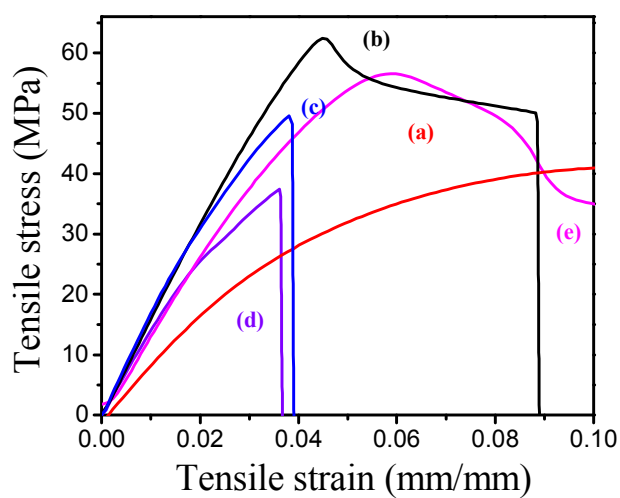

Figure S1. Partial enlargement of strain-stress curves of (a) neat PVDF, (b) neat PLLA, (c) pristine PVDF/PLLA (50/50, w/w) blend and PVDF/PLLA blends with $3 \mathrm{wt} \%$ reactive (d) linear and (e) graft copolymers of various topological structures 
Table S1. Mechanical Properties of Neat PVDF, Neat PLLA, Pristine PVDF/PLLA (50/50, w/w) Blend and JNMs Compatibilized PVDF/PLLA Blends with 3 wt\% Reactive Copolymers of Various Topological Structures

\begin{tabular}{cccc}
\hline Samples & $\begin{array}{c}\text { Elongation at }^{\text {break }}{ }^{\mathrm{a}}(\%) \\
\text { Neat PVDF }\end{array}$ & Yeilding strength $^{\text {a }}(\mathrm{MPa})$ & Tensile modulus $^{\text {a }}(\mathrm{GPa})$ \\
Neat PLLA & $8.9 \pm 5.6$ & $48.1 \pm 3.5$ & $1.0 \pm 0.2$ \\
PVDF/PLLA (50/50) & $3.8 \pm 1.1$ & - & $1.9 \pm 0.1$ \\
PVDF/PLLA/RLC-10 (50/50/3) & $3.6 \pm 1.9$ & - & $1.7 \pm 0.3$ \\
PVDF/PLLA/RGC-10 (50/50/3) & $256 \pm 9.8$ & $56.7 \pm 5.1$ & $1.6 \pm 0.1$ \\
\hline
\end{tabular}

${ }^{\mathrm{a}}$ calculated from tensile measurement. 


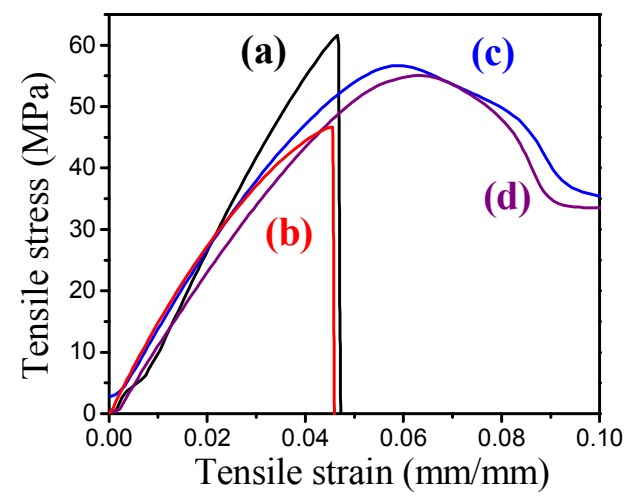

Figure S2. Partial enlargement of stress-strain curves of JNMs compatibilized PVDF/PLLA/RGC $(50 / 50 / 3, \mathrm{w} / \mathrm{w} / \mathrm{w})$ blends with various epoxy content: RGC-0, RGC-5, RGC-10 and RGC-20 represented epoxy of (a) $0 \mathrm{wt} \%$, (b) $5 \mathrm{wt} \%$, (c) $10 \mathrm{wt} \%$ and (d) $20 \mathrm{wt} \%$ in RGC, respectively 


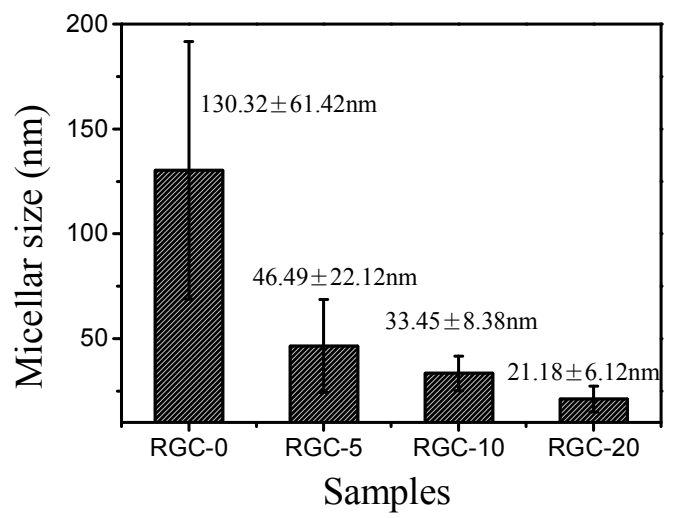

Figure S3. The TEM statistical analysis of nanomicelles at the PVDF-PLLA interface of its compatibilized PVDF/PLLA (50/50, w/w) blends with various epoxy content: RGC-0, RGC-5, RGC-10 and RGC-20 represented epoxy of $0 w t \%, 5 w t \%, 10 w t \%$ and $20 w t \%$ in $\mathrm{RGC}$, respectively 
Table S2. Thermal Parameters and Mechanical Properties of JNMs Compatibilized PVDF/PLLA/RGC (50/50/3, w/w/w) Blends with Various Epoxy Contents in RGC

\begin{tabular}{|c|c|c|c|c|c|c|c|}
\hline \multirow{2}{*}{ Samples } & \multicolumn{2}{|c|}{ DSC data } & \multicolumn{3}{|c|}{ DMA data } & \multirow{2}{*}{$\begin{array}{c}\text { Elongation at } \\
\text { break }^{\mathrm{c}}(\%)\end{array}$} & \multirow{2}{*}{$\begin{array}{c}\text { Yeilding } \\
\text { strength }^{\mathrm{c}}(\mathrm{MPa})\end{array}$} \\
\hline & $T_{g, P L L A}{ }^{\mathrm{a}}\left({ }^{\circ} \mathrm{C}\right)$ & $T_{m, P L L A}{ }^{\mathrm{a}}\left({ }^{\circ} \mathrm{C}\right)$ & $T_{g, P V D F}{ }^{\mathrm{b}}\left({ }^{\circ} \mathrm{C}\right)$ & $T_{g, P L L A}{ }^{\mathrm{b}}\left({ }^{\circ} \mathrm{C}\right)$ & $\Delta T_{g}{ }^{\mathrm{b}}\left({ }^{\circ} \mathrm{C}\right)$ & & \\
\hline PVDF/PLLA/RGC-0 $(50 / 50 / 3)^{\mathrm{d}}$ & 64.8 & $169.8,163.7$ & -37.5 & 75.2 & 112.7 & $4.5 \pm 3.9$ & $61.6 \pm 3.9$ \\
\hline PVDF/PLLA/RGC-5 (50/50/3) ${ }^{\mathrm{d}}$ & 63.7 & 165.22 & -34.7 & 74.6 & 109.3 & $5 \pm 3.1$ & $46.5 \pm 6.1$ \\
\hline PVDF/PLLA/RGC-10 (50/50/3) ${ }^{\mathrm{d}}$ & 62.9 & $165.8,162.6$ & -36.4 & 72.5 & 108.9 & $256 \pm 9.8$ & $56.7 \pm 5.1$ \\
\hline PVDF/PLLA/RGC-20 $(50 / 50 / 3)^{\mathrm{d}}$ & 61.8 & $164.7,161.6$ & -37.5 & 69.1 & 106.6 & $318 \pm 19.7$ & $55.1 \pm 5.3$ \\
\hline
\end{tabular}

${ }^{\mathrm{a}}$ calculated from the second heating scanning curves in the DSC data; ${ }^{\mathrm{b}}$ obtained from the DMA data; ${ }^{c}$ calculated from tensile measurement; ${ }^{\mathrm{d}}$ RGC-0, RGC-5, RGC-10 and RGC-20 represented epoxy of $0 \mathrm{wt} \%, 5 \mathrm{wt} \%, 10 \mathrm{wt} \%$ and $20 \mathrm{wt} \%$ in $\mathrm{RGC}$, respectively. 


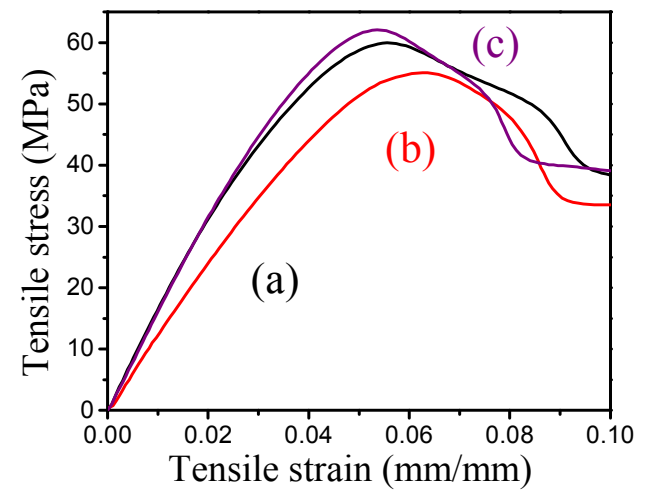

Figure S4. Partial enlargement of stress-strain curves of JNMs compatibilized PVDF/PLLA $(50 / 50, w / w)$ blends with various GRC-20 loadings ((a) $1 \mathrm{wt} \%$, (b) $3 \mathrm{wt} \%$ and (c) $5 \mathrm{wt} \%)$. 
Table S3. Mechanical Properties of JNMs Compatibilized PVDF/PLLA (50/50, w/w) Blends with Various GRC-20 Loadings (0wt\%, 1wt\%, 3wt\% and 5wt\%)

\begin{tabular}{|c|c|c|}
\hline Samples & $\begin{array}{c}\text { Elongation at } \\
\text { break }^{\mathrm{a}}(\%)\end{array}$ & Yeilding strength $^{\mathrm{a}}(\mathrm{MPa})$ \\
\hline PVDF/PLLA/RGC-20 (50/50/0) & $3.8 \pm 3.1$ & - \\
\hline PVDF/PLLA/RGC-20 (50/50/1) & $242 \pm 12.9$ & - \\
\hline PVDF/PLLA/RGC-20 (50/50/3) & $318 \pm 19.7$ & $55.1 \pm 5.3$ \\
\hline PVDF/PLLA/RGC-20 (50/50/5) & $380 \pm 16.9$ & $62.1 \pm 6.9$ \\
\hline
\end{tabular}

${ }^{\mathrm{a}}$ calculated from tensile measurement 


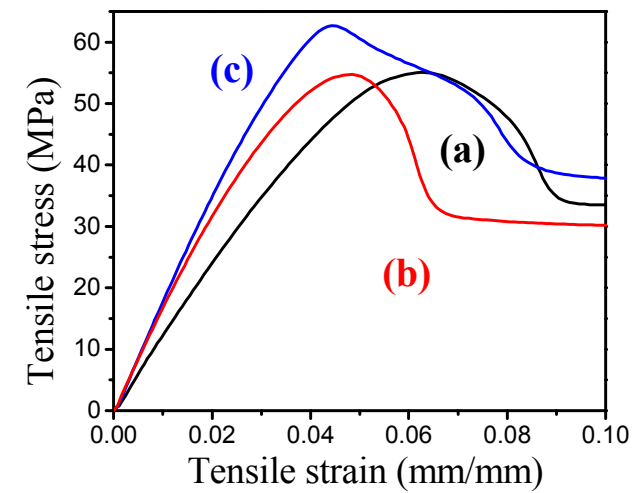

Figure S5. Partial enlargement of stress-strain curves of PVDF/PLLA/RGC-20 (50/50/3, w/w/w) blend with various mixing methods (a) simultaneous mixing, (b) premixing with PVDF and (c) premixing with PLLA 
Table S4. Mechanical Properties of JNMs Compatibilized PVDF/PLLA/RGC-20 (50/50/3, w/w/w) Blends with Various Mixing Methods

\begin{tabular}{ccc}
\hline Samples & $\begin{array}{c}\text { Elongation at } \\
\text { break }^{\mathrm{a}}(\%)\end{array}$ & Yeilding strength $^{\mathrm{a}}(\mathrm{MPa})$ \\
\hline Simultaneous mixing & $318 \pm 19.7$ & $55.2 \pm 9.9$ \\
PVDF-premixed & $380 \pm 16.9$ & $54.7 \pm 6.8$ \\
PLLA-premixed & $67 \pm 9.8$ & $62.7 \pm 8.9$ \\
\hline
\end{tabular}

${ }^{a}$ calculated from tensile measurement 
Table S5. Molecular Parameters of Components in Blends

\begin{tabular}{ccccc} 
Code & Sample & $M_{n}{ }^{\mathrm{a}} / \times 10^{4}$ & $M_{w}{ }^{\mathrm{a}} / \times 10^{4}$ & $P D I^{\mathrm{a}}$ \\
\hline 1 & PVDF & 10.5 & 21 & 2.0 \\
2 & PLLA-H & 8.93 & 15.8 & 1.77 \\
3 & PLLA-M & 6.2 & 13.4 & 2.16 \\
4 & PLLA-H & 0.15 & 0.79 & 5.29 \\
\hline${ }^{\mathrm{a}} M_{n}, M_{w}$ and $P D I$ determined by GPC
\end{tabular}

